\title{
Sensitizing solid tumors to CAR-mediated cytotoxicity using synthetic antigens
}

\section{Lena Gamboa}

Georgia Institute of Technology

Ali Zamat

Georgia Institute of Technology

\section{Daryll Vanover}

Georgia Institute of Technology

Chloé Thiveaud

Georgia Institute of Technology

Hannah Peck

Georgia Institute of Technology

Hathaichanok Phuengkham

Georgia Institute of Technology

Anirudh Sivakumar

Georgia Institute of Technology https://orcid.org/0000-0003-0788-6476

Adrian Harris

Georgia Institute of Technology

Shreyas Dahotre

Georgia Institute of Technology

Fang-Yi Su

Georgia Institute of Technology

Philip Santangelo

Georgia Institute of Technology https://orcid.org/0000-0001-7352-0339

Gabriel Kwong ( $\nabla$ gkwong@gatech.edu )

Georgia Institute of Technology https://orcid.org/0000-0002-6255-6755

Article

Keywords:

Posted Date: January 28th, 2022

DOI: https://doi.org/10.21203/rs.3.rs-1276779/v1 
License: (c) (i) This work is licensed under a Creative Commons Attribution 4.0 International License. Read Full License 


\section{Sensitizing solid tumors to CAR-mediated cytotoxicity using synthetic antigens}

Authors: Lena Gamboa ${ }^{1}$, Ali H. Zamat ${ }^{1}$, Daryll Vanover ${ }^{1}$, Chloé A. Thiveaud ${ }^{1}$, Hannah E. Peck ${ }^{1}$, Hathaichanok Phuengkham ${ }^{1}$, Anirudh Sivakumar ${ }^{1}$, Adrian M. Harris ${ }^{1}$, Shreyas N. Dahotre ${ }^{1}$, Fang-Yi Su ${ }^{1}$, Philip J. Santangelo ${ }^{1}$, Gabriel A. Kwong ${ }^{1-5,{ }^{*}}$

Affiliations:

1 Wallace H. Coulter Department of Biomedical Engineering, Georgia Institute of Technology and Emory School of Medicine, Atlanta, GA 30332

2 Parker H. Petit Institute of Bioengineering and Bioscience, Atlanta, GA 30332.

103 Institute for Electronics and Nanotechnology, Georgia Tech, Atlanta, GA 30332.

4 The Georgia Immunoengineering Consortium, Emory University and Georgia Tech, Atlanta, GA 30332.

5 Winship Cancer Institute, Emory University, Atlanta, GA 30332.

${ }^{*}$ Corresponding author. Email: gkwong@gatech.edu 


\begin{abstract}
CAR T cell immunotherapy relies on CAR targeting of tumor-associated antigens, yet heterogenous antigen expression, interpatient variation, and off-tumor expression by healthy cells remain barriers. Here, we develop synthetic antigens to sensitize solid tumors for recognition and

5 elimination by CAR T cells. Unlike tumor-associated antigens, we design synthetic antigens that are orthogonal to endogenous proteins to eliminate off-tumor targeting and that have a small genetic footprint to facilitate efficient tumor delivery to tumors by viral vectors. Using the RSV-F camelid single-domain antibody $(\mathrm{VHH})$ as a synthetic antigen, we show that adoptive transfer of a $\mathrm{VHH}$ CAR T cells to mice bearing $\mathrm{VHH}$ expressing tumors reduced tumor burden in multiple

10 syngeneic mouse models of cancer, improved survival, induced epitope spread, and protected against tumor rechallenge. Our work supports in situ delivery of synthetic antigens to treat antigen low or negative tumors with CAR T cells.
\end{abstract}




\section{MAIN TEXT}

Despite the therapeutic efficacy of chimeric antigen receptor (CAR) T cells to treat certain hematological malignancies ${ }^{1}$, their clinical success against solid tumors remains limited ${ }^{2-4}$. Identifying tumor-associated antigens (TAA) is particularly challenging ${ }^{5}$ as heterogenous antigen expression, low antigen density, and antigen loss reduce CAR T cell efficacy and raise concerns with antigen escape ${ }^{6-8}$. Expression of CAR antigens at low-levels in healthy tissue also pose safety concerns arising from on-target off-tumor toxicities ${ }^{9-11}$. aCD19 CAR T cell killing of healthy B cells, for example, results in hypogammaglobulinemia and cytopenia that requires lifelong IgG replacement therapy while also carrying the risk of neurotoxicity due to low-level expression of

10 CD19 on healthy brain mural cells ${ }^{12}$. T cells targeting solid tumor-associated antigens (TAAs) (e.g., HER2, CAIX) show cross-reactivity with healthy cells in vital organs ${ }^{4,13}$ that limit safe and effective clinical translation ${ }^{14}$. Emerging strategies to address the scarcity of tumor antigens include genome wide activation of endogenous genes using CRISPRa to bypass the need to identify TAAs ${ }^{15}$, combinatorial or universal antigen sensing circuits to enhance the ability of engineered $\mathrm{T}$ cells to discriminate malignant cells from normal tissue expressing a single epitope $^{16-20}$, or spatial control of CAR T cells to release bispecific T cell engagers to redirect T cells against tumor cells that do not express the CAR antigen ${ }^{21,22}$. To alleviate the need to validate CARs for each target antigen candidate, direct delivery of CD19 via oncolytic virus or GFP by tumor-colonizing bacteria enabled tumor recognition by a single CAR $\mathrm{T}$ cell construct ${ }^{23-25}$.

20 Collectively, these efforts underscore the need to develop strategies that reduce the bottleneck of antigen selection and enable CAR T cell recognition of tumors otherwise lacking targetable antigens.

We developed synthetic antigens to sensitize solid tumors to CAR T cell mediated immunity agnostic to a tumor's endogenous antigen expression profile. Ideally, a synthetic antigen

25 would be characterized by several key features: it should be (1) orthogonal to endogenous 
proteins to minimize off-tumor toxicity, (2) genetically encoded for delivery by viral and nonviral approaches, (3) small and compact to facilitate gene transfer, (4) stably expressed to facilitate recognition and (5) targetable by single-chain variable fragments (scFvs) to enable engineering of cognate CAR T cells against them. Considering these criteria, we repurposed a xenogeneic protein, the antigen binding fragment of the heavy-chain-only camelid antibodies $(\mathrm{VHH})$, as a synthetic antigen. Its small genetic footprint ( $\sim 375 \mathrm{bp})$, thermal stability, protease resistance, and stability at extreme $\mathrm{pH}$ have facilitated numerous in vivo applications ${ }^{26-28}$, including as neutralizing agents $^{29,30}$, fusion proteins for imaging ${ }^{31,32}$, payload carriers for targeted vaccine delivery ${ }^{33,34}$, and chimeric antigen receptors in lieu of the traditional scFv domain ${ }^{35}$. We demonstrate stable expression of $\mathrm{VHH}$ on the surface of various tumor types, including breast, colon, and lung cancer, following mRNA or AAV delivery, which subsequently led to recognition and killing by $\alpha \mathrm{VHH}$ CAR T cells. Moreover, we show $\alpha \mathrm{VHH}$ CARs are well tolerated in naïve C57BL/6J mice at the doses tested by serum chemistry. Adoptive transfer of $\alpha \mathrm{VHH}$ CAR T cells to mice bearing $\mathrm{VHH}$-treated tumors reduced tumor burden in multiple syngeneic models of cancer, improved survival, induced epitope spread and prevented tumor growth after rechallenge. Our data shows that sensitizing tumors to CAR-mediated cytotoxicity using synthetic antigens could potentiate antitumor immunity and improve responses against solid tumors.

\section{RESULTS}

To design synthetic antigens for CAR targeting, we tested two xenogeneic molecules, SunTag and VHH. SunTag is a repeat of the 19-aa epitope EELLSKNYHLENEVARLKK of the yeast transcriptional factor GCN4, which has previously been integrated into a protein tagging system due to its small genetic footprint, orthogonality to naturally occurring sequences in the 25 genome, and ability to bind to its cognate scFv with high affinity and specificity ${ }^{36}$ while VHHs are 
small ( 15 kDa) antigen binding fragments derived from camelid antibodies and are highly stable in vivo ${ }^{26-28}$. To identify a genetic construct with high and sustained expression of surface-bound synthetic antigens, we designed and tested several synthetic antigen constructs comprised of a membrane anchor for surface expression and retention, a linker to provide flexibility to access the 5 antigen domain, and either a SunTag or VHH antigen domain for CAR T cell recognition (Fig. 1A). We first compared the CD4 transmembrane (CD4TM) domain ${ }^{37}$ to the glycosylphosphatidylinositol (GPI) anchor from the decay accelerating factor (DAF) ${ }^{37-39}$ as membrane anchors for $\mathrm{VHH}$ against the human respiratory syncytial virus (RSV) F protein as a model synthetic antigen ${ }^{40}$. The CD4TM domain functions as a transmembrane anchor that tethers

10 fused proteins to the plasma membrane, such as split GFP for single-molecule imaging ${ }^{37}$ and $\mathrm{CARs}^{41,42}$. Similarly, GPI attachment to the C-terminus of a protein is a post-translational modification that anchors proteins to the upper leaflet of the plasma membrane $e^{43,44}$, thus enabling the attachment of synthetic proteins to the cell surface including split proteins ${ }^{37}$, nanobodies ${ }^{30}$, and costimulatory molecules ${ }^{45}$. To compare both membrane anchors, we quantified $\mathrm{VHH}$ expression in wildtype E0771, MC38, and A549 cancer cells by surface staining and flow cytometry following mRNA transfection (Fig. 1B). Maximum surface expression, as measured by median fluorescence intensity (MFI), was $\sim 10$-fold higher for GPI-anchored VHH compared to CD4TM-anchored VHH across the three tumor cell lines (Fig. 1C). Moreover, VHH expression was maintained for 5-12 days for the GPI-anchored construct compared to 2-4 days for CD4TM-

$20 \mathrm{VHH}$ (Fig. 1C). Due to higher and sustained expression, we chose to use GPI as the membrane anchor for synthetic antigens.

Next, to evaluate SunTag and $\mathrm{VHH}$ as antigen domains, we compared the expression kinetics of GPI-anchored SunTag to GPI-anchored VHH. To determine the effect of linker length on synthetic antigen expression, we first designed two SunTag constructs, each linked to the GPI 25 anchor by either a $1 x$ repeat of the flexible G4S peptide or a $3 x$ repeat of G4S. Given that peptides 
are generally more susceptible to enzymatic or chemical degradation than proteins, we hypothesized that fusing SunTag to a protein could enhance its surface expression. Therefore, we designed a third construct where SunTag was fused to the $\mathrm{C}$ terminus of VHH. All three GPIanchored SunTag constructs were indistinguishable from background fluorescence by day 3 post

5 transfection in A549 cells (Fig. 1D), demonstrating that neither linker length nor fusion to $\mathrm{VHH}$ affected surface expression of SunTag. Moreover, while VHH-linked SunTag became undetectable by day 3 (Fig. 1D, triangles), the $\mathrm{VHH}$ linker (and not its tethered SunTag) remained detectable on $96.7 \%$ of cells by flow cytometry 5 days post transfection (Fig. S1), showing that VHH expression is sustained on the surface longer than SunTag. To confirm this, we further tested VHH expression across E0771, MC38, and A549 tumor lines and found half-maximal expression for GPI-anchored, 1xG4S-linked SunTag was reached on average 1.8 days post transfection, while $\mathrm{VHH}$ reached half-maximal expression on average by $4.8 \mathrm{~d}$ (Fig. 1D). Altogether, GPIanchored $\mathrm{VHH}$ showed sustained surface expression on multiple cell lines, both murine and human, for at least 5 days (Fig. 1E, Fig. S1-S2). For comparison, the Kb-SIINFEKL pMHC complex has a half-life of $\sim 8$ hrs on the surface of MC38 cells (Fig. S3), while various human immune cells such as DCs, B cells, and monocytes have a reported half-life of the HLA-A*02:01gp100154-162 complex of $1.5-22.5 \mathrm{hrs}^{46}$.

\section{aSunTag and aVHH CARs recognize and kill tumor cells expressing their cognate}

\section{0 synthetic antigen in vitro}

To target synthetic antigens, we designed murine CARs where the anti-VHH and antiGCN4 scFv sequences were fused to the murine CD8 $\alpha$ hinge and transmembrane domain, CD28 costimulatory domain, and the $\mathrm{CD} 3 \zeta$ signaling domains (Fig. 2A-B). Similarly, human CARs comprised either an anti-VHH or anti-GCN4 scFv, followed by the human CD8 $\alpha$ hinge and

25 transmembrane domain, 4-1BB costimulatory domain, and CD3ろ cytolytic domain (Fig. S4A). 
Both $\alpha \mathrm{VHH}$ and aSunTag CARs were stably expressed on the surface of both primary murine and human T cells following viral transduction (Fig. 2C, Fig. S4B). To test antigen-dependent activation, we co-incubated murine anti-VHH or anti-SunTag CAR T cells with E0771 breast cancer cells transfected with either $\mathrm{VHH}$ or SunTag (Fig. 2D) and observed significant

5 upregulation of CD25 and CD69 activation markers in greater than 94\% of CAR T cells following exposure to their cognate synthetic antigen (Fig. 2E). To assess CAR T cell cytotoxicity, $\alpha \mathrm{VHH}$ and $\alpha$ SunTag CAR T cells were co-cultured at a 2:1 effector:target cell ratio, $\alpha \mathrm{VHH}$ and $\alpha$ SunTag CAR T cells produced interferon gamma (IFN-y) at significantly higher levels over wildtype T cells when co-cultured with $\mathrm{VHH}$ - or SunTag-expressing tumor cells $\left({ }^{* * *} \mathrm{p}<0.001\right)$, respectively. By contrast, no significant elevation was detected when cultured alone or with tumor cells expressing a mismatched synthetic antigen (Fig. 2F). Similarly, we observed significantly elevated levels of IFN-y secretion when human CAR T cells were co-incubated with their cognate synthetic antigens (Fig. S4C-D).

Next, we assessed the ability of both $\alpha \mathrm{VHH}$ and aSunTag CARs to kill tumor cells expressing their cognate synthetic antigen. At a 2:1 effector to target ratio, no cytolytic activity was observed by untransduced T cells on either SunTag- or VHH-transfected E0771 tumor cells or by CAR T cells cultured on tumor cells expressing a mismatched synthetic antigen (i.e., aSunTag CAR T cells on VHH-transfected tumor cells, aVHH CAR T cells on SunTag-transfected tumor cells). When $\alpha \mathrm{VHH}$ and aSunTag CAR $\mathrm{T}$ were co-incubated with their cognate synthetic antigen, however, we observed significant increases in cytotoxicity for both murine $\left({ }^{* * * *} p<0.001\right.$, aVHH CAR: $63.2 \%$, aST CAR: $46.6 \%$, Fig. 2 G) and human CAR T cells $\left({ }^{* * * *} p<0.001\right.$, aVHH CAR: 71.3\%, aST CAR: $43.7-51.0 \%$, Fig. S4E-F). Both murine and human VHH CAR T cells killed target cells more potently than their SunTag CAR T cell counterparts (Fig. 2G, Fig. S4E-F), consistent with earlier data which showed higher secretion of IFN-y by aVHH CAR T cells (Fig.

25 2F, Fig. S4C-D). Collectively, our data show that tumor cells expressing synthetic antigens can 
be recognized and killed by CAR T cells. Based on these data, combined with the sustained expression of $\mathrm{VHH}$, we selected the $\mathrm{VHH}$ synthetic antigen and $\alpha \mathrm{VHH} \mathrm{CAR}$ T cells for subsequent in vivo validation.

aVHH CAR T cells enhance antitumor immunity against solid tumors

A key concern with antigen selection and the development of CAR T cells against them is toxicity in off-tumor tissues ${ }^{4}$. We postulated that $\alpha \mathrm{VHH}$ CAR T cells would exhibit minimal offtumor toxicity since its target antigen is not natively found in mice. To test this, we adoptively transferred primary murine $\mathrm{aVHH}$ CAR $\mathrm{T}$ cells by intravenous (i.v.) infusion into

10 immunocompetent and naïve C57BL6/J mice and collected serum 7 days post transfer for analysis. Compared to saline controls or mice receiving an equal number of untransduced T cells, we found no significant elevations in serum chemistry values, including total protein, aspartate transaminase (AST), alanine aminotransferase (ALT), urea nitrogen (BUN), creatinine, phosphorous, and calcium (Fig. 3A). Additionally, no significant difference was observed in body weight between naïve mice receiving saline, wildtype T cells, or aVHH CAR T cells (Fig. S5). These data showed that $\mathrm{QVHH}$ CAR T cells were well tolerated systemically, with no appreciable abnormalities in renal and liver function nor in body weight. Furthermore, due to their low immunogenicity $^{47,48}$, we did not expect expression of the $\mathrm{VHH}$ antigen alone to alter tumor growth. To test this, we compared tumor growth kinetics of wildtype E0771 and MC38 tumors with tumor cells transduced to stably express $\mathrm{VHH}$ and found that $\mathrm{VHH}$ expression did not alter tumor growth in either tumor model (Fig. S6). These data show that $\mathrm{VHH}$ expression is not sufficient to reduce tumor burden.

To evaluate the antitumor efficacy in vivo, we adoptively transferred $\alpha \mathrm{VHH}$ CAR T cells into immunocompetent mice bearing syngeneic MC38 colon adenocarcinoma tumors that were 25 transduced to express the $\mathrm{VHH}$ synthetic antigen. Compared to treatment with control aSunTag 
CAR T cells, transfer of $\alpha \mathrm{VHH}$ CAR T cells significantly reduced tumor burden (Fig. 3B, C); within 18 days of adoptive cell transfer, tumor volumes within the control group grew on average 9-fold, whereas those treated with $\alpha \mathrm{VHH}$ CAR T cells grew $\sim 2.5$-fold. Flow cytometric analysis of the tumor, spleen, and the ipsilateral (i.e., tumor draining) and contralateral lymph nodes revealed

5 that $\alpha \mathrm{VHH}$ CAR $\mathrm{T}$ cell treatment increased the number of CD8+ tumor infiltrating lymphocytes (TILs) compared to treatment with control CAR T cells $\left({ }^{* *} p<0.01\right.$, Fig. 3D). Additionally, CAR+ CD8 $T$ cells comprised a higher frequency of the CD8+ TILs within mice receiving $\alpha \mathrm{VHH}$ CAR T cells $\left(55 \%\right.$ vs. $22 \%,{ }^{* *} p<0.01$, Fig. $\left.3 D\right)$.

Therapies that lead to immunogenic cell death promote the release and cross-

10 presentation of tumor antigens in the draining lymph nodes and can prime de novo $\mathrm{T}$ cell responses, analogous to vaccination ${ }^{49-51}$. By using the tumor as a source of antigens, in situ vaccination strategies, such as the intratumoral delivery of IL-12-encoding RNA ${ }^{52}$ or combination treatment with FIt3L, radiotherapy, and TLR3 agonist ${ }^{53}$, result in epitope spread that increase response rates to therapy. To determine whether targeting the $\mathrm{VHH}$ synthetic antigen would result

15 in priming against tumor antigens, we quantified the percentage of endogenous T cells reactive against Reps1, an H-2Db-restricted mutant neoepitope previously characterized in MC38 cells ${ }^{54}$. In the tumors of $a \mathrm{VHH}$ CAR $\mathrm{T}$ cell treated mice, we observed a significant increase in accumulation of CAR-negative, Reps1-reactive CD8+ T cells in the tumors $\left({ }^{*} p<0.05\right.$, Fig. 3D) as well as in the ipsilateral lymph nodes $\left({ }^{* * *} p<0.01\right.$, Fig. $\left.3 E\right)$. In tumor bearing mice treated with

20 control CAR T cells, however, we found no significant difference in the frequency of Reps1reactive $T$ cells between the ipsilateral and contralateral lymph nodes (Fig. 3E). These data provide evidence that CAR-mediated killing of $\mathrm{VHH}+$ tumors primes endogenous $\mathrm{T}$ cells against tumor antigens and promotes epitope spread.

We further sought to evaluate antitumor efficacy of $\alpha \mathrm{VHH}$ CAR $\mathrm{T}$ cells in a different 25 preclinical model of mice bearing syngeneic triple negative breast cancer (TNBC). TNBC tumors 
lack estrogen and progesterone receptor (ER/PR) as well as HER2 expression, making them unresponsive to endocrine and anti-HER2 therapies ${ }^{55,56}$. We therefore tested $\alpha \mathrm{VHH}$ CAR T cells in mice bearing E0771 tumors, a TNBC originally isolated from a spontaneous mammary tumor in a female C57BL/6 mouse ${ }^{57}$. Following adoptive transfer of $\alpha \mathrm{VHH}$ CAR T cells (Fig 4A), 3 of 4

5 mice treated with aVHH CAR T cells were complete responders for at least 100 days after ACT $(C R=3 / 4$, blue traces, Fig. 4B), while all those which received control CARs reached endpoint criteria (tumor volume $>1,000 \mathrm{~mm} 3$ ) within 30 days of treatment $(C R=0 / 4$, black traces, Fig. 4B). Furthermore, to determine whether treatment could initiate epitope spread and provide protection against the wildtype tumor, either naïve mice or complete responders were rechallenged with wildtype VHH-negative E0771 tumor cells in the contralateral mammary fat pad 45 days after initial therapy (Fig. 4A). While naïve mice grew tumors that reached endpoint in 28-32 days, complete responders rechallenged with $\mathrm{VHH}$-negative E0771 tumors were resistant to tumor growth $\left({ }^{* * *} p<0.0001\right.$, Fig. 4 C). Additionally, survival of rechallenged mice was significantly extended by at least 70 days over mice treated with control CAR T cells $\left({ }^{* *} p<0.01\right.$, Fig. 4D).

15 Collectively, these data demonstrate potent antitumor activity of $\alpha \mathrm{VHH}$ CAR T cells in two distinct syngeneic murine tumor models, as well as their potential to elicit antitumor responses analogous to in situ vaccination.

\section{AAV-mediated expression of VHH followed by treatment with aVHH CAR T cells leads to a} potent antitumor response

We next sought to explore whether in situ delivery of synthetic antigens could sensitize wildtype tumors to CAR T cell cytotoxicity (Fig. 4E). Several different viral and nonviral delivery systems are available for effective gene delivery in vivo ${ }^{58}$. Here, we used an adeno-associated viral (AAV) vector containing GPI-anchored $\mathrm{VHH}$ driven by a CMV promoter (AAV2-VHH), as well

25 as a control vector expressing firefly luciferase (AAV2-Fluc), to enable direct delivery of the $\mathrm{VHH}$ 
synthetic antigen to tumors. To assess $\mathrm{VHH}$ expression on tumor cells following AAV delivery, we injected either PBS, AAV2-Fluc or AAV2-VHH intratumorally into MC38 and E0771 tumors expressing a Thy1.1 reporter and quantified $\mathrm{VHH}$ expression by flow cytometry staining of Thy1.1+ cells. We found that AAV2-mediated delivery of $\mathrm{VHH}$ led to the expression of $\mathrm{VHH}$ on

5 the surface of tumor cells (E0771 $=57.3 \% \mathrm{VHH}+, \mathrm{MC} 38=37.9 \% \mathrm{VHH}+$, Fig. 4F, Fig. S7). These results were consistent with immunofluorescence analysis of tumor sections from mice bearing wildtype E0771 (E0771-wt) tumors injected intratumorally with either AAV2-VHH or AAV2-Fluc, further confirming $\mathrm{VHH}$ expression in AAV2-VHH treated tumors (Fig. 4G). Finally, we combined AAV delivery of the $\mathrm{VHH}$ synthetic antigen with systemic administration of $\alpha \mathrm{VHH}$ CAR T cells.

10 Established E0771-wt tumors were first treated with an i.t. injection of PBS, AAV2-Fluc, or AAV2VHH followed by an i.v. injection of $\alpha \mathrm{VHH}$ CAR T cells (Fig. 4E). Whereas treatment with AAV2Fluc $+\alpha$ VHH CAR T cells did not significantly affect tumor growth kinetics compared to PBS + aVHH CAR T cell controls, we observed treatment with AAV2-VHH + aVHH CAR T cells led to a significant delay in tumor growth $\left({ }^{* * *} \mathrm{p}<0.0001\right.$, Fig. $\left.4 \mathrm{H}\right)$. Likewise, we observed a significant reduction in tumor burden for wildtype MC38 tumors only in the case where mice were treated i.t. AAV2-VHH + i.v. aVHH CAR T cells (Fig. S8). These data demonstrate that delivery of the VHH synthetic antigen combined with $\alpha \mathrm{VHH}$ CAR T cell therapy sensitizes wildtype tumors to immune recognition and significantly improves antitumor responses in vivo.

\section{DISCUSSION}

The scarcity of tumor-specific target antigens limits the safety and efficacy of CAR T cell therapies for solid tumors. Here we developed synthetic antigens to sensitize solid tumors for recognition and elimination by CAR T cells. Unlike tumor-associated antigens, we designed a synthetic antigen $\mathrm{VHH}$ that is orthogonal to endogenous proteins to eliminate off-tumor targeting

25 and that has a small genetic footprint to facilitate efficient delivery to tumors by viral vectors. To 
tether $\mathrm{VHH}$ to the outer surface of tumor cells, we found that the use of the GPI membrane anchor linked to a CAR recognition domain led to stable expression of $\mathrm{VHH}$ on the surface of various tumor types, including breast, colon, and lung cancer, following mRNA or AAV delivery. To provide $\mathrm{T}$ cells the ability to recognize $\mathrm{VHH}$-expressing tumors, we designed and validated $\mathrm{a} \mathrm{VHH}$

5 CAR T cells, which showed potent antigen-dependent activation, cytokine production, and cytotoxicity upon recognition of $\mathrm{VHH}$ on the surface of tumor cells in vitro. Importantly, $\alpha \mathrm{VHH}$ CAR T cells exhibited potent antitumor activity in vivo against $\mathrm{VHH}+$ tumors in syngeneic models of colorectal and TNBC, which led to recruitment of endogenous tumor-reactive cells, resistance to tumor rechallenge, and extended survival.

The vast majority of tumor antigens that are under evaluation as CAR targets are also expressed at low levels in healthy tissue. Consequently, on-target off-tumor toxicity with CAR T cells remains a primary safety concern including severe respiratory distress, hearing loss, vestibular dysfunction, liver toxicity, and neurotoxicity ${ }^{12,13,59-62}$. As a synthetic antigen derived from camelids, $\mathrm{VHH}$ is not expressed by healthy tissue. Our data demonstrates that $\mathrm{aVHH}$ CAR T cells are well tolerated in vivo, as measured by serum chemistry and body weights of non-tumor bearing mice. Nonetheless, preclinical murine models may not fully recapitulate CAR T cell cytotoxicity in humans ${ }^{63}$ and thus, as with any new CAR T cell construct under consideration, future studies are warranted to assess the potential off-tumor activity of aVHH CAR T against healthy human tissue. Notably, however, the compact size, low immunogenicity, and stability of

20 VHHs shown in numerous preclinical studies ${ }^{26-28,30-35}$ as well as its FDA-approval as a neutralizing agents against the von Willebrand Factor ${ }^{29}$ further support that this class of molecules has the potential for safe clinical translation.

In addition to challenges in identifying solid tumor antigens with therapeutic potential, strategies that increase the therapeutic index of CARs by optimizing CAR affinity ${ }^{64,65}$, as well as 25 reports demonstrating the effects of linker length and intracellular domains on CAR function ${ }^{66,67}$, highlight the need to carefully optimize CAR constructs for each potential antigen target. This 
iterative process is costly and time-consuming, limiting downstream clinical implementation of CAR T cells for a broad range of tumor types. Moving forward, streamlining the CAR design process by enabling the use of a single optimized CAR construct against diverse tumors could improve the applicability of CAR T cell therapies for solid tumors. We envision that tumor delivery

5 of synthetic antigens could sensitize tumors for killing by CAR T cells as a primary mechanism and also secondarily 'kickstart' endogenous antitumor immunity - analogous to in situ vaccination strategies such as TLR agonists, radiation therapy, or cytokines that are used to prime or enhance anti-tumor immune responses ${ }^{68-71}$. Our results complement emerging strategies to deliver T cell targets to tumor cells including antibody-mediated delivery of immunodominant viral epitopes ${ }^{72}$,

10 intratumoral delivery of sfGFP using tumor colonizing bacteria (E. coli Nissle 1917$)^{24}$, and the local delivery of CD19 using oncolytic virus (OV), for subsequent treatment with their cognate CAR T cells ${ }^{23,25}$. In our studies, we observed that CAR T cell clearance of tumors expressing the VHH synthetic antigen primed endogenous $\mathrm{T}$ cells against neoantigens, as indicated by the increased number of Reps1 tetramer positive T cells in the tumor and draining lymph nodes in 15 the syngeneic MC38 murine model of colon adenocarcinoma. We also observed indication of epitope spread in mice bearing TNBC tumors where complete responders to $\alpha \mathrm{VHH}$ CAR T cell therapy were resistant to rechallenge to wildtype tumor cells that did not express the synthetic antigen, indicating the establishment of anti-tumor immunity against tumor antigens that were not targeted by CAR T cells. Although we used AAVs in this study for tumor delivery of $\mathrm{VHH}$, there is 20 an increasing number of FDA-approved viral (e.g., the oncolytic virus T-VEC ${ }^{73}$ ) and non-viral (e.g., mRNA-loaded lipid nanoparticles ${ }^{74,75}$ ) gene delivery vehicles amenable for intratumoral or locoregional delivery. Altogether, our studies provide support for the design and use of synthetic antigens to promote CAR-mediated tumor cytotoxicity for solid tumors with otherwise limited antigen targets. 


\section{ACKNOWLEDGEMENTS}

We thank Dr. D.R. Meyers (Emory) and C.D. Sago for helpful insights. This work was funded by the NIH Director's New Innovator Award (DP2HD091793), the National Center for Advancing Translational Sciences (UL1TR000454), the Shurl and Kay Curci Foundation, the NIH Shared

5 Instrumentation Grant (1S10OD016264-01A1) and was partially performed at the Georgia Tech Institute for Nanotechnology, a member of the National Nanotechnology Coordinated Infrastructure which is supported by the National Science Foundation (Grant ECCS-1542174). L.G. was supported by the Alfred P. Sloan Foundation and the National Institutes of Health GT BioMAT Training Grant under Award Number 5T32EB006343. L.G., A.S., and S.N.D. were supported by the National Science Foundation Graduate Research Fellowship under Grant No. DGE-1451512. G.A.K. holds a Career Award at the Scientific Interface from the Burroughs Wellcome Fund. F.S. was supported by a postdoctoral fellowship from the Wallace H. Coulter Department of Biomedical Engineering and the College of Engineering at Peking University. This content is solely the responsibility of the authors and does not necessarily represent the official views of the National Institutes of Health.

Competing interests: G.A.K. is co-founder of Glympse Bio and Port Therapeutics, and consults for Glympse Bio, Port Therapeutics, and Satellite Bio. This study could affect his personal financial status. The terms of this arrangement have been reviewed and approved by Georgia Tech in accordance with its conflict-of-interest policies. L.G., A.Z., D.V., P.J.S. and G.A.K. are listed as inventors on a patent application pertaining to the results of the paper. The patent applicant is the Georgia Tech Research Corporation. The application number is PCT/US21/54972. 


\section{FIGURES}

A

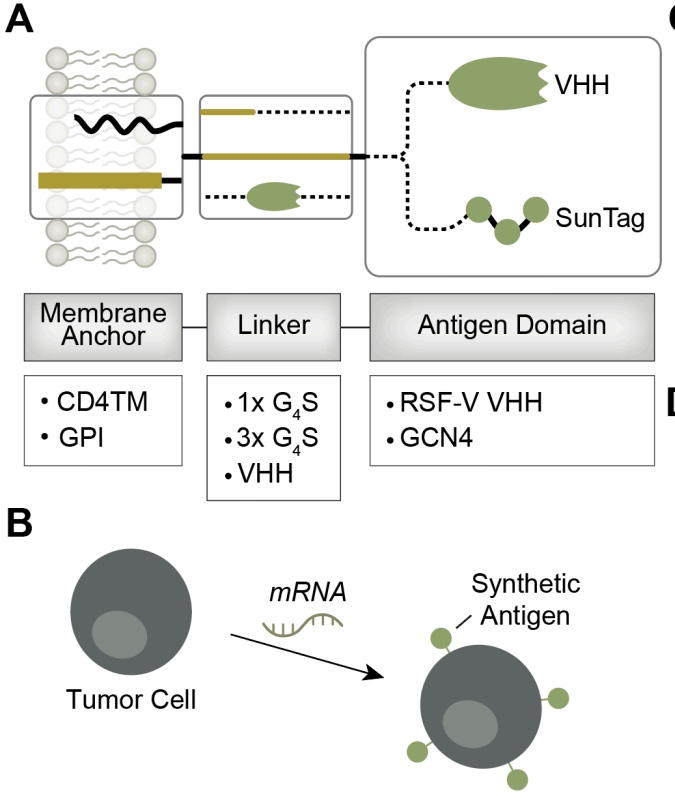

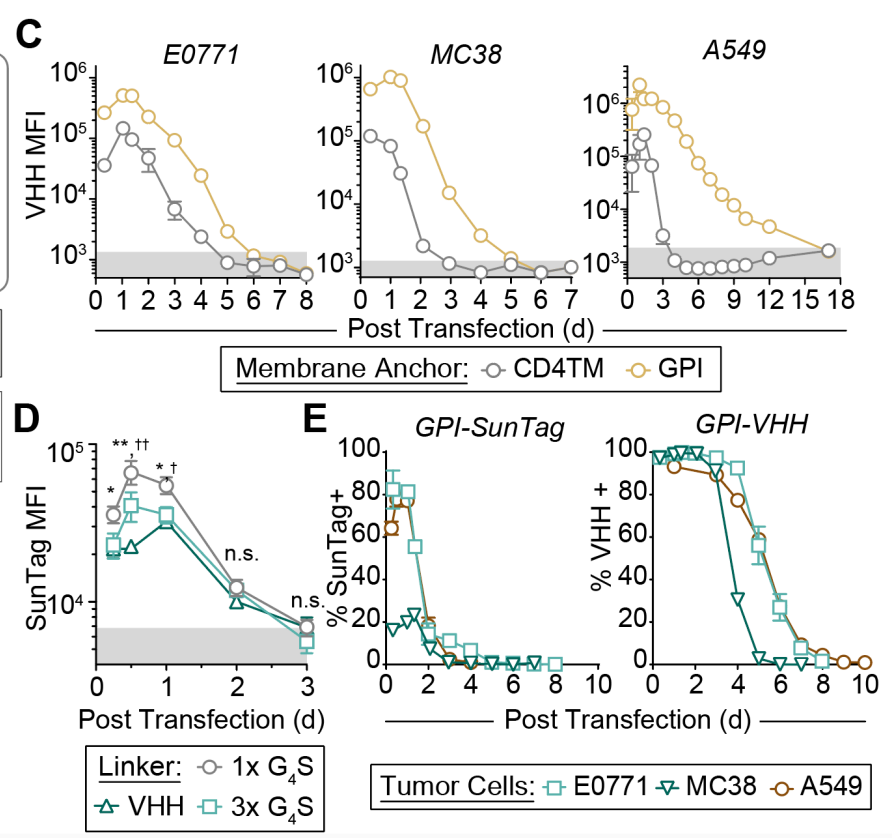

Fig. 1. Design of synthetic antigen constructs. (A) mRNA constructs tested consist of a membrane anchor, a linker, and a recognition domain. These constructs are (B) transfected into tumor cells to achieve synthetic antigen expression. (C) Expression kinetics of GPIanchored and CD4TM-anchored synthetic antigens consisting of a 1x G4S linker and the RSV$\mathrm{F} \mathrm{VHH} \mathrm{recognition} \mathrm{domain} \mathrm{on} \mathrm{the} \mathrm{surface} \mathrm{of} \mathrm{indicated} \mathrm{tumor} \mathrm{cells.} \mathrm{(D)} \mathrm{Expression} \mathrm{kinetics} \mathrm{of}$ mRNA constructs with a GPI-anchor, SunTag recognition domain, and indicated linker domains. Two-way ANOVA, mean \pm s.d. is depicted; $n=4$ (for $1 x$ G4S vs $3 x$ G4S: * $p<0.05$, ${ }^{* *} p<0.01$; for $1 \times$ G4S vs 3x G4S: $† p<0.05$, †† $p<0.01$ ). (E) Expression kinetics of GPIanchored SunTag or VHH on the surface of indicated tumor cell lines. 


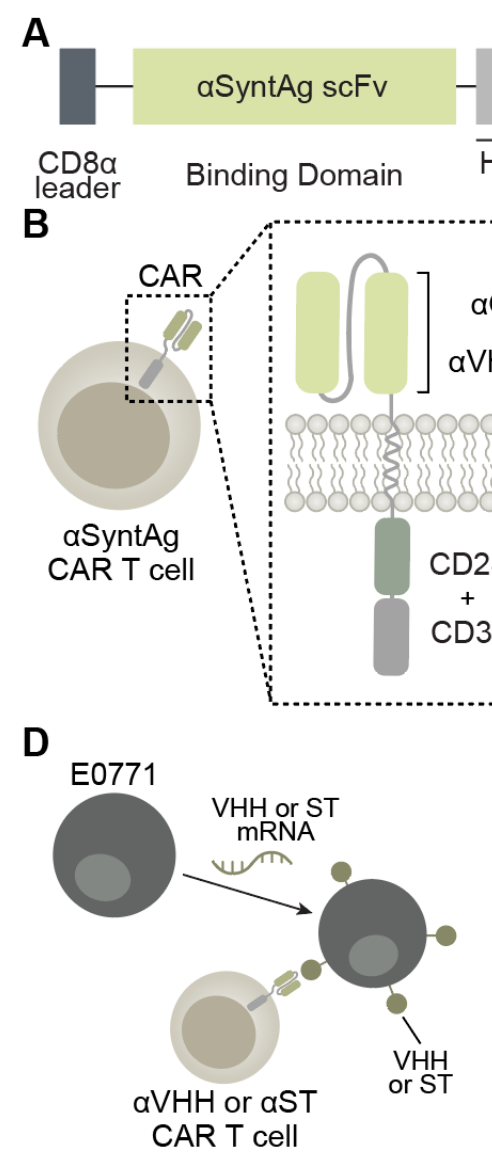

E
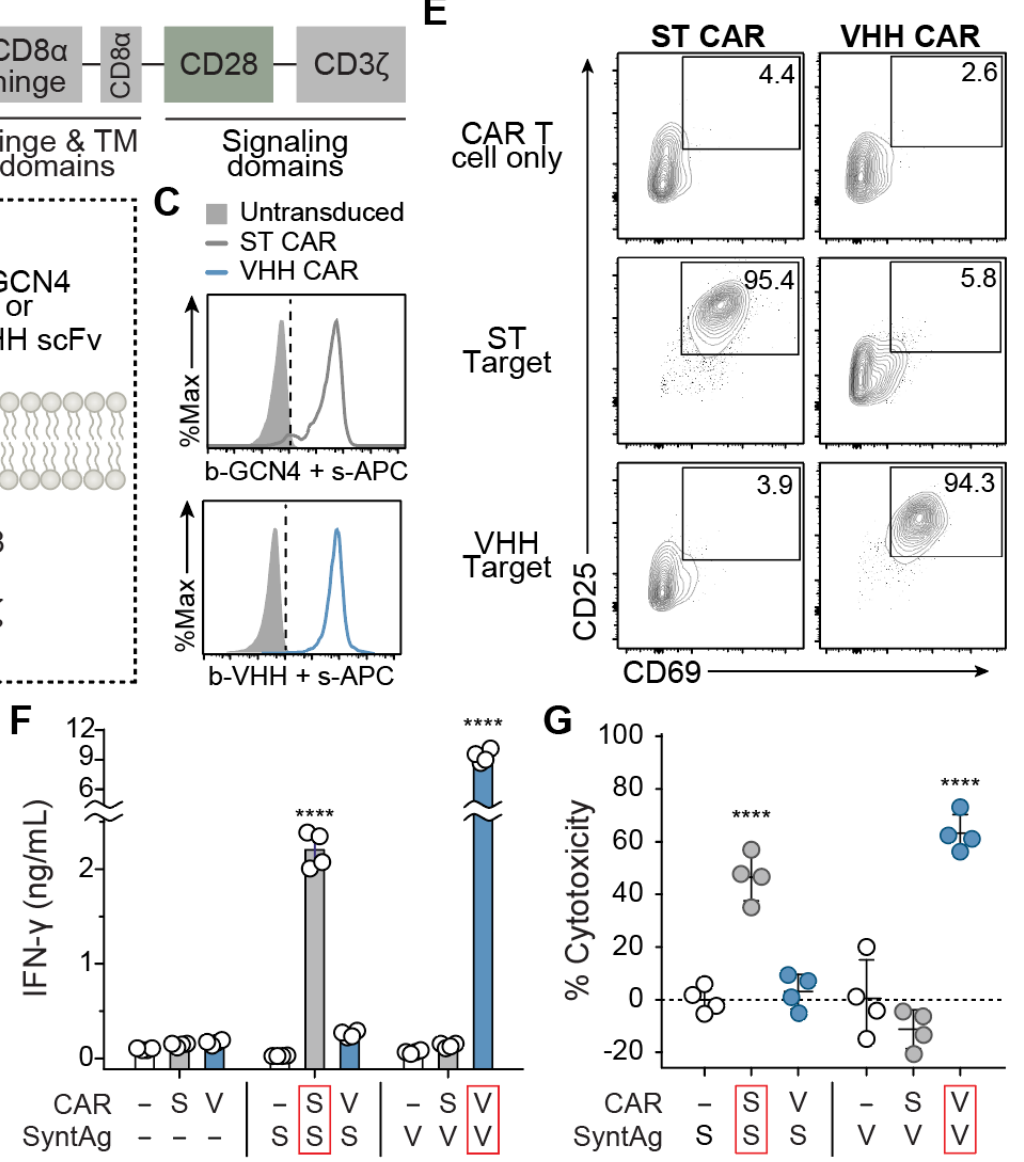

Fig. 2. Murine $\alpha \mathrm{VHH}$ and $\alpha \mathrm{GCN} 4 \mathrm{CAR} T$ cells recognize and kill tumor cells expressing cognate synthetic antigens. (A-B) Schematic of murine CAR constructs for targeting synthetic antigens (SyntAg) expressed on the surface of tumor cells (C) Surface expression of SunTag CAR (top) and VHH CAR (bottom) on primary murine T cells following retroviral transduction. (D) E0771 tumor cells were transfected with $\mathrm{VHH}$ or SunTag mRNA and co-incubated with either aVHH or aSunTag CARs. (E) Staining of indicated T cell population with activation markers CD25 and CD69 following co-incubation with ST- or VHH-expressing E0771 tumor cells. (F) Interferon gamma (IFN-y) secretion by murine aSunTag CAR T, aVHH CAR T or untransduced (WT) T cells following a $24 \mathrm{hr}$ co-culture at a 2:1 effector:target (E:T) ratio with E0771 transfected with either VHH or SunTag mRNA. (G) Killing of transfected E0771 tumor cells following the same 24-hr co-culture. One-way ANOVA; mean \pm s.d. is depicted; $n=4 ;{ }^{* * * *} p<0.0001$. 


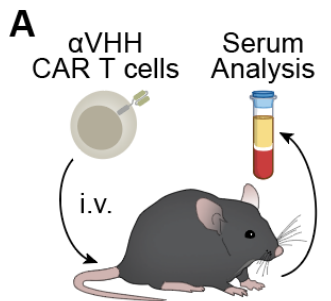

Naïve C57BL6/J
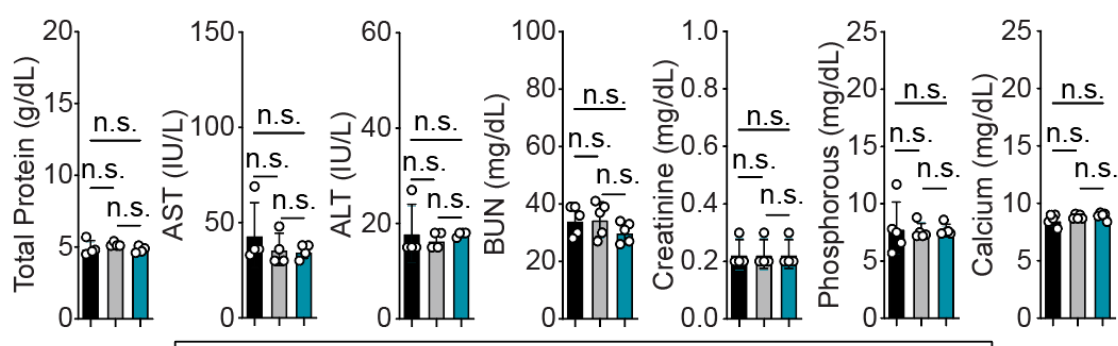

Saline

$\square$ Untransduced T cells

$\square$ aVHH CAR T cells

B

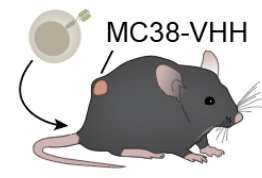

Inoculation

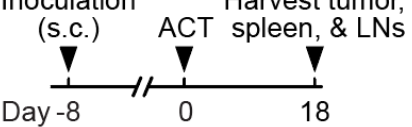

Analyze T cell Populations:

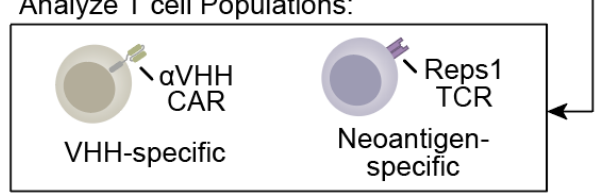

D

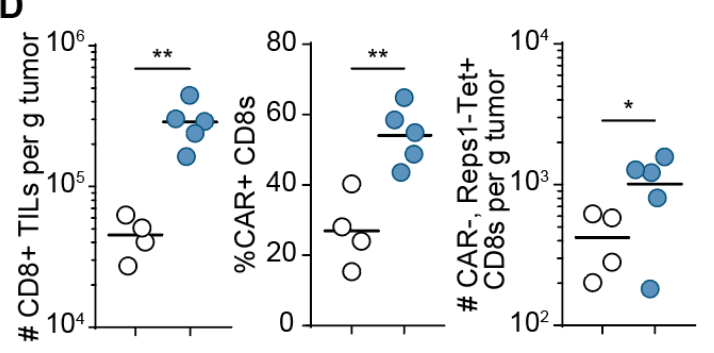

O Ctrl CAR T cells $\bigcirc$ aVHH CAR T cells

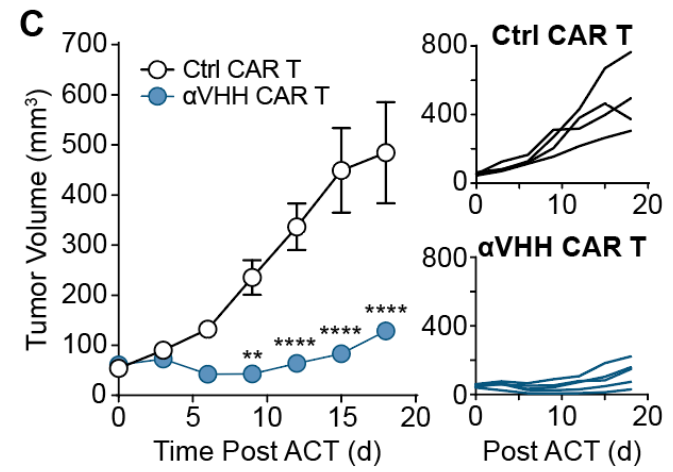

E

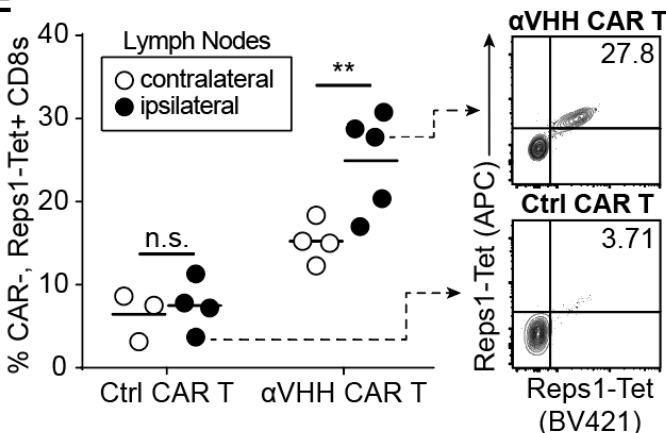

Fig. 3. Adoptive transfer of $a \mathrm{VHH}$ CAR $\mathrm{T}$ cells into mice with $\mathrm{VHH}$-expressing tumors delays tumor growth, promotes infiltration of tumor-reactive $\mathrm{T}$ cells in the tumor, and leads to an increased frequency of tumor reactive T cells in the lymph nodes. (A) Blood serum analysis $7 d$ post i.v. administration of aVHH CAR T cells, untransduced $\mathrm{T}$ cells, or saline into naïve C57BL6/J mice. One-way ANOVA; mean \pm s.d. is depicted; $n=4 ; n . s .=$ not significant (B) Mice bearing MC38-VHH tumors were treated with aVHH CAR T cells. (C) Tumor growth curves of MC38-VHH tumor-bearing mice treated with aVHH CAR T cells. Two-way ANOVA, mean \pm s.e.m. is depicted; $n=4$. (D) Count and frequency of indicated T cell populations isolated from the tumor. Unpaired t-test with Welch's correction for unequal variance was performed (left and right panels); unpaired Student's t-test (middle panel). (E) Representative flow plot of 
endogenous (CAR-) T cell expression of the Reps1 TCR in tdLNs and the frequency of this population in the ipsilateral and contralateral draining lymph nodes. Unpaired Student's t-test, mean is depicted; $n=4-5 ;{ }^{*} p<0.05 ;{ }^{* *} p<0.01 ;{ }^{* *} p<0.001 ;{ }^{* * *} p<0.0001$. 
A
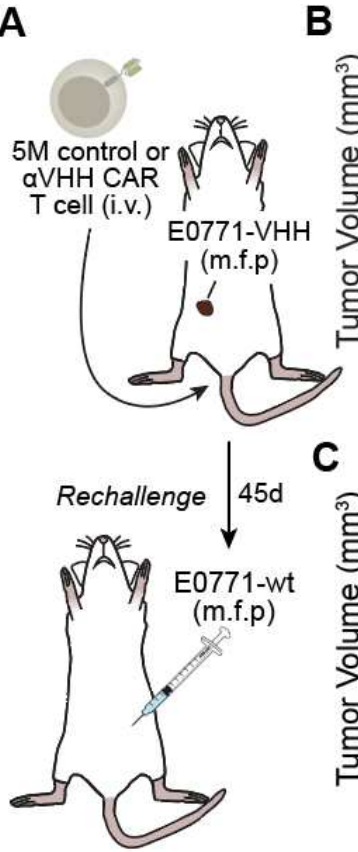

D

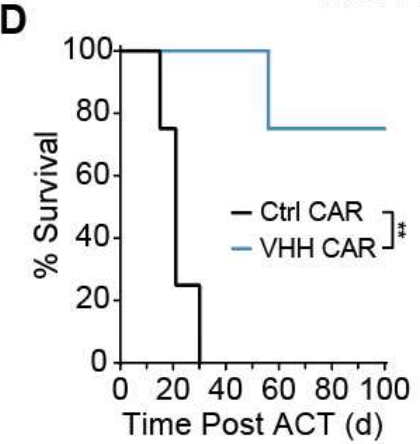

E

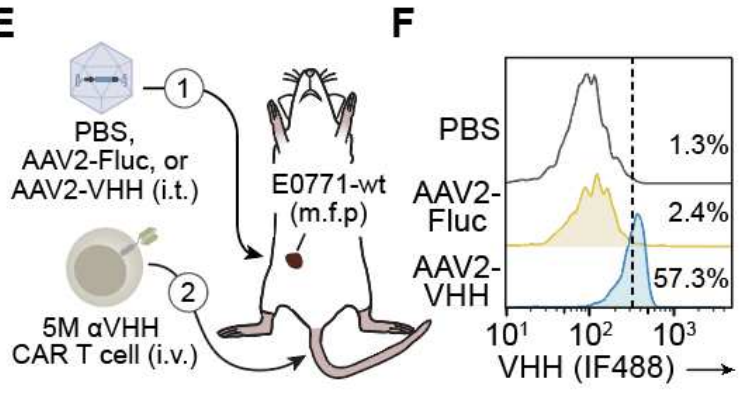

G
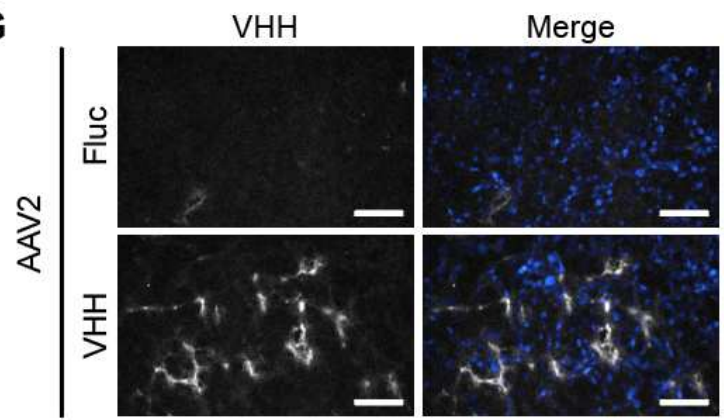

H

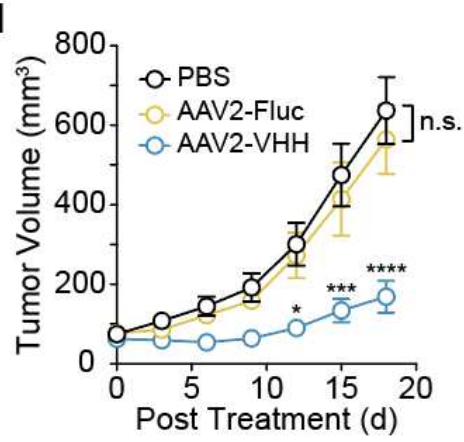

Fig. 4. Synthetic antigen treatment promotes potent antitumor immunity in an immunocompetent model of triple negative breast cancer. (A) Mice bearing E0771-VHH tumors were treated with aVHH CAR T cells. (B) Individual traces of tumor growth curves of E0771VHH tumor-bearing mice treated with control CAR T cells (black) or aVHH CAR T cells (blue) $(C R=$ complete responders $) .45$ days after initial treatment, cured mice were $(C)$ rechallenged with wildtype E0771 tumor cells. VHH expression on wildtype or transduced MC38 and E0771 tumor cells. Two-way ANOVA, mean \pm s.e.m. is depicted; n.s. = not significant. (D) Survival curves of tumor-bearing mice following initial treatment and rechallenge, log-rank (Mantel-Cox) test; ${ }^{* *} p<0.01$. (E) Mice bearing wildtype E0771 tumors were first treated with AAV2-Fluc or 
AAV2-VHH, followed by adoptive transfer of $\alpha \mathrm{VHH}$ CAR T cells. (F) Flow cytometry analysis of VHH expression on E0771-Thy1.1 dissociated tumor cells 48 hrs following AAV treatment of $6 e 9$ GCs. Plot is gated on Thy1.1+ cells. (G) Tumor cryosections were fixed and stained for VHH expression. Scale bar $=60 \mu \mathrm{m} .(\mathrm{H})$ Tumor growth curves of wildtype E0771 tumor-bearing mice treated with AAV2 (Fluc or VHH) and $\alpha \mathrm{VHH}$ CAR T cells. Two-way ANOVA, mean \pm s.e.m. is depicted; $n=6 ;{ }^{*} p<0.05 ;{ }^{* * *} p<0.001 ;{ }^{* * *} p<0.0001$. 


\section{REFERENCES}

\section{REFERENCES}

1 Maude, S. L. et al. Tisagenlecleucel in Children and Young Adults with B-Cell Lymphoblastic Leukemia. New England Journal of Medicine 378, 439-448, doi:10.1056/NEJMoa1709866 (2018).

2 Lim, W. A. \& June, C. H. The Principles of Engineering Immune Cells to Treat Cancer. Cell 168, 724-740, doi:10.1016/j.cell.2017.01.016 (2017).

3 Rafiq, S., Hackett, C. S. \& Brentjens, R. J. Engineering strategies to overcome the current roadblocks in CAR T cell therapy. Nature Reviews Clinical Oncology 17, 147-167, doi:10.1038/s41571-019-0297-y (2020).

4 Hou, A. J., Chen, L. C. \& Chen, Y. Y. Navigating CAR-T cells through the solid-tumour microenvironment. Nature Reviews Drug Discovery 20, 531-550, doi:10.1038/s41573021-00189-2 (2021).

155 Martinez, M. \& Moon, E. K. CAR T Cells for Solid Tumors: New Strategies for Finding, Infiltrating, and Surviving in the Tumor Microenvironment. Frontiers in Immunology 10, doi:10.3389/fimmu.2019.00128 (2019).

6 Majzner, R. G. \& Mackall, C. L. Tumor Antigen Escape from CAR T-cell Therapy. Cancer Discovery 8, 1219-1226, doi:10.1158/2159-8290.Cd-18-0442 (2018).

207 Majzner, R. G. et al. Tuning the Antigen Density Requirement for CAR T-cell Activity. Cancer Discov 10, 702-723, doi:10.1158/2159-8290.Cd-19-0945 (2020).

8 Hamieh, M. et al. CAR T cell trogocytosis and cooperative killing regulate tumour antigen escape. Nature 568, 112-116, doi:10.1038/s41586-019-1054-1 (2019).

9 Wudhikarn, K. et al. Infection during the first year in patients treated with CD19 CAR T cells for diffuse large B cell lymphoma. Blood Cancer Journal 10, 79, doi:10.1038/s41408-020-00346-7 (2020).

10 Nahas, G. et al. Persistent Cytopenias after Chimeric Antigen Receptor T-Cell Immunotherapy for CD19+ Aggressive Lymphoma: A Single Institution Experience. Biology of Blood and Marrow Transplantation 25, S180,

11 Fried, S. et al. Early and late hematologic toxicity following CD19 CAR-T cells. Bone Marrow Transplantation 54, 1643-1650, doi:10.1038/s41409-019-0487-3 (2019).

12 Parker, K. R. et al. Single-Cell Analyses Identify Brain Mural Cells Expressing CD19 as Potential Off-Tumor Targets for CAR-T Immunotherapies. Cell 183, 126-142.e117, doi:10.1016/j.cell.2020.08.022 (2020).

13 Morgan, R. A. et al. Case report of a serious adverse event following the administration of T cells transduced with a chimeric antigen receptor recognizing ERBB2. Mol Ther 18, 843-851, doi:10.1038/mt.2010.24 (2010).

14 Ramakrishna, S., Barsan, V. \& Mackall, C. Prospects and challenges for use of CAR T cell therapies in solid tumors. Expert Opinion on Biological Therapy 20, 503-516, doi:10.1080/14712598.2020.1738378 (2020).

15 Wang, G. et al. Multiplexed activation of endogenous genes by CRISPRa elicits potent antitumor immunity. Nature Immunology 20, 1494-1505, doi:10.1038/s41590-019-05004 (2019).

4516 Cho, J. H., Collins, J. J. \& Wong, W. W. Universal Chimeric Antigen Receptors for Multiplexed and Logical Control of T Cell Responses. Cell 173, 1426-1438.e1411, doi:10.1016/j.cell.2018.03.038 (2018). 
17 Roybal, Kole T. et al. Precision Tumor Recognition by T Cells With Combinatorial Antigen-Sensing Circuits. Cell 164, 770-779, doi:https://doi.org/10.1016/j.cell.2016.01.011 (2016).

18 Lohmueller, J. J., Ham, J. D., Kvorjak, M. \& Finn, O. J. mSA2 affinity-enhanced biotinbinding CAR T cells for universal tumor targeting. Oncoimmunology 7, e1368604, doi:10.1080/2162402x.2017.1368604 (2017).

19 Lee, Y. G. et al. Use of a Single CAR T Cell and Several Bispecific Adapters Facilitates Eradication of Multiple Antigenically Different Solid Tumors. Cancer Research 79, 387396, doi:10.1158/0008-5472.Can-18-1834 (2019).

20 Landgraf, K. E. et al. convertibleCARs: A chimeric antigen receptor system for flexible control of activity and antigen targeting. Communications Biology 3, 296, doi:10.1038/s42003-020-1021-2 (2020).

21 Miller, I. C. et al. Enhanced intratumoural activity of CAR T cells engineered to produce immunomodulators under photothermal control. Nature Biomedical Engineering, doi:10.1038/s41551-021-00781-2 (2021).

22 Choi, B. D. et al. CAR-T cells secreting BiTEs circumvent antigen escape without detectable toxicity. Nature Biotechnology 37, 1049-1058, doi:10.1038/s41587-019-01921 (2019).

23 Park, A. K. et al. Effective combination immunotherapy using oncolytic viruses to deliver CAR targets to solid tumors. Science Translational Medicine 12, eaaz1863, doi:doi:10.1126/scitranslmed.aaz1863 (2020).

24 Vincent, R. L. et al. Probiotic-guided CAR-T cells for universal solid tumor targeting. bioRxiv, 2021.2010.2010.463366, doi:10.1101/2021.10.10.463366 (2021).

25 Aalipour, A. et al. Viral Delivery of CAR Targets to Solid Tumors Enables Effective Cell Therapy. Molecular Therapy - Oncolytics 17, 232-240, doi:https://doi.org/10.1016/j.omto.2020.03.018 (2020).

26 Hussack, G., Hirama, T., Ding, W., Mackenzie, R. \& Tanha, J. Engineered single-domain antibodies with high protease resistance and thermal stability. PLoS One 6, e28218, doi:10.1371/journal.pone.0028218 (2011).

27 Kunz, P. et al. The structural basis of nanobody unfolding reversibility and thermoresistance. Scientific Reports 8, 7934, doi:10.1038/s41598-018-26338-z (2018).

28 Ingram, J. R., Schmidt, F. I. \& Ploegh, H. L. Exploiting Nanobodies' Singular Traits. Annual Review of Immunology 36, 695-715, doi:10.1146/annurev-immunol-042617053327 (2018).

29 Peyvandi, F. et al. Caplacizumab for Acquired Thrombotic Thrombocytopenic Purpura. New England Journal of Medicine 374, 511-522, doi:10.1056/NEJMoa1505533 (2016).

30 Tiwari, P. M. et al. Engineered mRNA-expressed antibodies prevent respiratory syncytial virus infection. Nat Commun 9, 3999, doi:10.1038/s41467-018-06508-3 (2018).

31 Movahedi, K. et al. Nanobody-Based Targeting of the Macrophage Mannose Receptor for Effective <em $>$ In Vivo</em $>$ Imaging of Tumor-Associated Macrophages. Cancer Research 72, 4165-4177, doi:10.1158/0008-5472.Can-11-2994 (2012).

32 Keyaerts, M. et al. Phase I Study of $<$ sup $>68</$ sup $>$ Ga-HER2-Nanobody for PET/CT Assessment of HER2 Expression in Breast Carcinoma. Journal of Nuclear Medicine 57, 27-33, doi:10.2967/jnumed.115.162024 (2016). 
33 Nchinda, G. et al. The efficacy of DNA vaccination is enhanced in mice by targeting the encoded protein to dendritic cells. J Clin Invest 118, 1427-1436, doi:10.1172/jci34224 (2008).

34 Idoyaga, J. et al. Comparable T helper 1 (Th1) and CD8 T-cell immunity by targeting HIV gag p24 to CD8 dendritic cells within antibodies to Langerin, DEC205, and Clec9A. Proc Natl Acad Sci U S A 108, 2384-2389, doi:10.1073/pnas.1019547108 (2011).

35 Xie, Y. J. et al. Nanobody-based CAR T cells that target the tumor microenvironment inhibit the growth of solid tumors in immunocompetent mice. Proceedings of the National Academy of Sciences 116, 7624-7631, doi:10.1073/pnas.1817147116 (2019). Tanenbaum, M. E., Gilbert, L. A., Qi, L. S., Weissman, J. S. \& Vale, R. D. A proteintagging system for signal amplification in gene expression and fluorescence imaging. Cell 159, 635-646, doi:10.1016/j.cell.2014.09.039 (2014).

37 Pinaud, F. \& Dahan, M. Targeting and imaging single biomolecules in living cells by complementation-activated light microscopy with split-fluorescent proteins. Proceedings of the National Academy of Sciences 108, E201-E210, doi:10.1073/pnas.1101929108 (2011).

38 Du, Y., Pattnaik, A. K., Song, C., Yoo, D. \& Li, G. Glycosyl-phosphatidylinositol (GPI)anchored membrane association of the porcine reproductive and respiratory syndrome virus GP4 glycoprotein and its co-localization with CD163 in lipid rafts. Virology 424,

39 Nickells, M. W., Alvarez, J. I., Lublin, D. M. \& Atkinson, J. P. Characterization of DAF2, a high molecular weight form of decay-accelerating factor (DAF; CD55), as a covalently cross-linked dimer of DAF-1. J Immunol 152, 676-685 (1994).

40 Rossey, I. et al. Potent single-domain antibodies that arrest respiratory syncytial virus fusion protein in its prefusion state. Nat Commun 8, 14158-14158, doi:10.1038/ncomms14158 (2017).

41 Brown, C. E. et al. Optimization of IL13R $\alpha 2$-Targeted Chimeric Antigen Receptor T Cells for Improved Anti-tumor Efficacy against Glioblastoma. Molecular therapy : the journal of the American Society of Gene Therapy 26, 31-44, doi:10.1016/j.ymthe.2017.10.002 (2018).

42 Dotti, G., Gottschalk, S., Savoldo, B. \& Brenner, M. K. Design and development of therapies using chimeric antigen receptor-expressing T cells. Immunol Rev 257, 107-126, doi:10.1111/imr.12131 (2014).

43 Ferguson, M. A. \& Williams, A. F. Cell-surface anchoring of proteins via glycosylphosphatidylinositol structures. Annu Rev Biochem 57, 285-320, doi:10.1146/annurev.bi.57.070188.001441 (1988).

44 Medof, M. E., Nagarajan, S. \& Tykocinski, M. L. Cell-surface engineering with GPIanchored proteins. Faseb j 10, 574-586, doi:10.1096/fasebj.10.5.8621057 (1996).

45 McHugh, R. S., Nagarajan, S., Wang, Y.-C., Sell, K. W. \& Selvaraj, P. Protein Transfer of Glycosyl-phosphatidylinositol-B7-1 into Tumor Cell Membranes. A Novel Approach to Tumor Immunotherapy 59, 2433-2437 (1999).

46 Zehn, D., Cohen, C. J., Reiter, Y. \& Walden, P. Extended presentation of specific MHCpeptide complexes by mature dendritic cells compared to other types of antigenpresenting cells. European Journal of Immunology 34, 1551-1560, doi:https://doi.org/10.1002/eji.200324355 (2004). 
47 Cortez-Retamozo, V. et al. Efficient tumor targeting by single-domain antibody fragments of camels. Int J Cancer 98, 456-462, doi:10.1002/ijc.10212 (2002).

48 Jovčevska, I. \& Muyldermans, S. The Therapeutic Potential of Nanobodies. BioDrugs 34, 11-26, doi:10.1007/s40259-019-00392-z (2020).

549 Brossart, P. The Role of Antigen Spreading in the Efficacy of Immunotherapies. Clinical Cancer Research 26, 4442-4447, doi:10.1158/1078-0432.Ccr-20-0305 (2020).

50 Heckler, M. \& Dougan, S. K. Unmasking Pancreatic Cancer: Epitope Spreading After Single Antigen Chimeric Antigen Receptor T-Cell Therapy in a Human Phase I Trial. Gastroenterology 155, 11-14, doi:10.1053/j.gastro.2018.06.023 (2018).

1051 Beatty, G. L. et al. Mesothelin-Specific Chimeric Antigen Receptor mRNA-Engineered T Cells Induce Antitumor Activity in Solid Malignancies. Cancer Immunology Research 2, 112-120, doi:10.1158/2326-6066.Cir-13-0170 (2014).

$52 \mathrm{Li}, \mathrm{Y}$. et al. Multifunctional oncolytic nanoparticles deliver self-replicating IL-12 RNA to eliminate established tumors and prime systemic immunity. Nature Cancer 1, 882-893,

53 Hammerich, L. et al. Systemic clinical tumor regressions and potentiation of PD1 blockade with in situ vaccination. Nature Medicine 25, 814-824, doi:10.1038/s41591019-0410-x (2019).

54 Yadav, M. et al. Predicting immunogenic tumour mutations by combining mass spectrometry and exome sequencing. Nature 515, 572-576, doi:10.1038/nature14001 (2014).

55 Yin, L., Duan, J.-J., Bian, X.-W. \& Yu, S.-c. Triple-negative breast cancer molecular subtyping and treatment progress. Breast Cancer Research 22, 61, doi:10.1186/s13058020-01296-5 (2020).

2556 Dees, S., Ganesan, R., Singh, S. \& Grewal, I. S. Emerging CAR-T Cell Therapy for the Treatment of Triple-Negative Breast Cancer. Molecular Cancer Therapeutics 19, 24092421, doi:10.1158/1535-7163.Mct-20-0385 (2020).

57 Johnstone, C. N. et al. Functional and molecular characterisation of EO771.LMB tumours, a new C57BL/6-mouse-derived model of spontaneously metastatic mammary cancer. Disease Models \& Mechanisms 8, 237-251, doi:10.1242/dmm.017830 (2015).

58 Nayerossadat, N., Maedeh, T. \& Ali, P. A. Viral and nonviral delivery systems for gene delivery. Adv Biomed Res 1, 27-27, doi:10.4103/2277-9175.98152 (2012).

59 Seaman, B. J. et al. Audiovestibular dysfunction associated with adoptive cell immunotherapy for melanoma. Otolaryngol Head Neck Surg 147, 744-749, doi:10.1177/0194599812448356 (2012).

60 Lamers, C. H. et al. Treatment of metastatic renal cell carcinoma with CAIX CARengineered T cells: clinical evaluation and management of on-target toxicity. Molecular therapy : the journal of the American Society of Gene Therapy 21, 904-912, doi:10.1038/mt.2013.17 (2013).

$4061 \quad$ Nomura, N. et al. Prostate specific membrane antigen (PSMA) expression in primary gliomas and breast cancer brain metastases. Cancer Cell Int 14, 26, doi:10.1186/14752867-14-26 (2014).

62 Gust, J., Taraseviciute, A. \& Turtle, C. J. Neurotoxicity Associated with CD19-Targeted CAR-T Cell Therapies. CNS Drugs 32, 1091-1101, doi:10.1007/s40263-018-0582-9 (2018). 
63 Ruella, M. \& June, C. H. Predicting Dangerous Rides in CAR T Cells: Bridging the Gap between Mice and Humans. Mol Ther 26, 1401-1403, doi:10.1016/j.ymthe.2018.05.005 (2018).

64 Liu, X. et al. Affinity-Tuned ErbB2 or EGFR Chimeric Antigen Receptor T Cells Exhibit an Increased Therapeutic Index against Tumors in Mice. Cancer research 75, 3596-3607, doi:10.1158/0008-5472.CAN-15-0159 (2015).

65 Hernandez-Lopez, R. A. et al. T cell circuits that sense antigen density with an ultrasensitive threshold. Science 371, 1166-1171, doi:doi:10.1126/science.abc1855 (2021).

1066 Singh, N. et al. Single Chain Variable Fragment Linker Length Regulates CAR Biology and T Cell Efficacy. Blood 134, 247-247, doi:10.1182/blood-2019-131024 (2019).

67 Sterner, R. C. \& Sterner, R. M. CAR-T cell therapy: current limitations and potential strategies. Blood Cancer Journal 11, 69, doi:10.1038/s41408-021-00459-7 (2021).

68 Marabelle, A., Tselikas, L., de Baere, T. \& Houot, R. Intratumoral immunotherapy: using the tumor as the remedy. Annals of Oncology 28, xii33-xii43, doi:https://doi.org/10.1093/annonc/mdx683 (2017).

69 Melero, I., Castanon, E., Alvarez, M., Champiat, S. \& Marabelle, A. Intratumoural administration and tumour tissue targeting of cancer immunotherapies. Nature Reviews Clinical Oncology 18, 558-576, doi:10.1038/s41571-021-00507-y (2021).

2070 Hong, W. X. et al. Intratumoral Immunotherapy for Early-stage Solid Tumors. Clinical Cancer Research 26, 3091-3099, doi:10.1158/1078-0432.Ccr-19-3642 (2020).

71 Hammerich, L., Binder, A. \& Brody, J. D. In situ vaccination: Cancer immunotherapy both personalized and off-the-shelf. Mol Oncol 9, 1966-1981, doi:10.1016/j.molonc.2015.10.016 (2015).

2572 Millar, D. G. et al. Antibody-mediated delivery of viral epitopes to tumors harnesses CMV-specific T cells for cancer therapy. Nature Biotechnology 38, 420-425, doi:10.1038/s41587-019-0404-8 (2020).

73 Johnson, D. B., Puzanov, I. \& Kelley, M. C. Talimogene laherparepvec (T-VEC) for the treatment of advanced melanoma. Immunotherapy 7, 611-619, doi:10.2217/imt.15.35 (2015).

74 Kaufman, H. L., Kohlhapp, F. J. \& Zloza, A. Oncolytic viruses: a new class of immunotherapy drugs. Nature Reviews Drug Discovery 14, 642-662, doi:10.1038/nrd4663 (2015).

75 Kowalski, P. S., Rudra, A., Miao, L. \& Anderson, D. G. Delivering the Messenger: Advances in Technologies for Therapeutic mRNA Delivery. Mol Ther 27, 710-728, doi:10.1016/j.ymthe.2019.02.012 (2019). 


\section{Supplementary Files}

This is a list of supplementary files associated with this preprint. Click to download.

- NatCancerSASupplementaryInformation.pdf 